\title{
Color discrimination thresholds and the Einstein's field equations
}

\author{
L.D. Lozhkin ${ }^{1}$, O.V. Osipov ${ }^{1}$ \\ ${ }^{1}$ Povolzhskiy State University of Telecommunications and Informatics, Lev Tolstoy street 23, 443010, Samara, Russia
}

\begin{abstract}
The paper discusses on the development of strictly equal-contrast color scale. It is known that in the CIE 1931 (x, y) thresholds for color discrimination are represented by ellipses, which are used to characterize the equal-contrast of a color system. Different color systems are described by different values of ellipticity (the size of the MacAdam ellipses). The paper proposes a new approach to the creation of strictly equal-contrast color systems based on similar concepts of «the color horizon» in the colorimetry and «the event horizon» in general relativity (GR). It is proposed to use an equation similar to Einstein's field equations for the transformation of ellipses in a two-dimensional color pattern into circles and ellipsoids of rotation in three-dimensional model - in equal diameter balls. It is introduced a concept of a curvature of a color discrimination space and a tensor of a «color power». It is obtained a matrix equation from which the coefficients of transformation into strictly equal-contrast color system are determined.
\end{abstract}

Keywords: image processing; strictly equal-contrast color space; color locus; CIE colorimetric system; the metric tensor; curvature tensor; color tensor; basis of the moving frame

\section{Introduction}

Development of perceivable equal-contrast three-dimensional color scale would represent not only a great scientific achievement, but also proved to be helpful anyway. Its application would simplify the definition of colors and setting of color tolerances, bring clarity to the question of interpretation of the one-dimensional color scales for identification of some different colors, serve as a guide in the production of standard color patterns and assist in the selection of harmonious color combinations. Unfortunately, attempts to establish such a scale have not led to a significant success yet. On the contrary, they have confirmed the assumption that it is impossible to create this strictly equal-contrast three-dimensional scale. However, these attempts at least indicate there may be good enough approximation of an ideal equal-contrast color space. In this article there is will be continued the development of strongly equal-contrast color scales, and special attention will be given to the conclusion of numerical expressions for such scales.

If the observer is offered white, black and a set of gray color samples and asked to choose the one that equally differs from white and from black samples, he will face with a little difficulty because the assessment of the relative value of the two big color differences, eventually, based only on subjective impression. This is a special case of the color discrimination definition, which Newhall called by the method of color discrimination ratios [1]. However, the desired gray color can be determined based on the average assessment of several observers, the desired accuracy depends only on the number of observers and the number of assessments made by them. Then, the color range, which is between black and mid-gray, can be bisected, corresponding to you can do with an interval between white and mid-gray. Thus, the range from black to white forms equal-contrast lightness scale consisting of five equally spaced colors according to the subjective sensation. It was one of the methods used to determine the Munsellgray scale [2]. The method of converting the color locus, proposed in [3], is also of great interest.

\section{The object of the study}

In the early 40s of the last century it were appeared the publications of the results of experiments MacAdam carried out to ascertain the color discrimination thresholds [4]. Thresholds of color discrimination were graphically displayed in the form of ellipses on the color chart CIE 1931 ( $x, y)$. Fig. 1 shows the results of MacAdam experiments which later became known as the MacAdam ellipses. In CIE 1931 ( $x, y$ ) color discrimination thresholds are shown by ellipses that can be used as a characteristic of the equal-contrast of a specific color system. It is sufficient to introduce the concept of color surface ellipticity defined as the ratio of the major to the minor axes of the ellipse. For various CIE colorimetric systems values of ellipticity are different [5].

Carry out a thought experiment. Reducing the brightness of the stimulus, at some point retinal cones with lower sensitivity, compared with sticks, switch off and there comes a scotopic vision in shades of gray, which means that the color discrimination threshold is increased and at zero brightness the color discrimination threshold is equal to infinity, exactly limited by color locus. It is obvious that an increase in the emission brightness to very high values leads to color sensitivity eyes will also decrease. This phenomenon can be explained by the fact that the collapse of iodopsin (photosensitive material of retinal cones) will be faster than his recovery. So the eye will be color-blind, i.e. the value of color discrimination threshold will increase with the brightness of radiation and at very high brightness the color discrimination threshold is equal to infinity. Graphically, this is shown in fig. $2 b$ as a hyperboloid of one sheet.

First of all, introduce some concepts:

1. The color horizon (similar to «the event horizon» in general relativity) is a volume in which, from the point of view of an eye color discrimination, color is homogeneous.

2. Infinity is the area bounded by the color locus. 


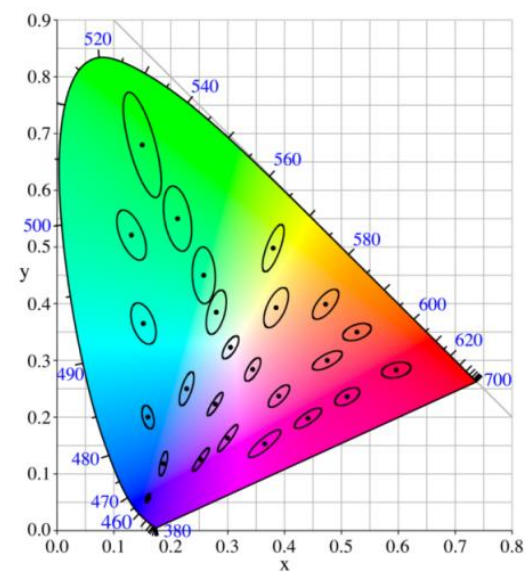

Fig. 1. The MacAdam ellipses (ellipses dimensions for clarity increased 10 times).

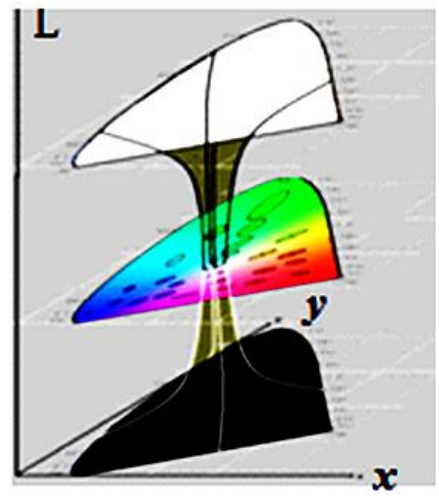

Fig. 2. Dependence of the color discrimination thresholdson the brightness.

Then consider some areas of the hyperboloid. The upper and lower portions, colored respectively in white and black colors, it can be said that the horizon of color extend to infinity. These surfaces are linear, flat and have a Euclidean geometry. A more complex structure is space located in the central part of the hyperboloid.

The horizon of color has a small radius and consequently the space enclosed by the horizon color is curved and closed, similarly as it occurs in the fundamental theory of stellar evolution. In connection with this it is possible to solve the problems of color discrimination thresholds as well as to create new equal-contrast color systems, in which all MacAdam ellipses would be transformed into equal circles and in three dimensions - in equal diameter balls, using Einstein's field equations [6].

\section{Methods}

Einstein field equations for relativistic gravitation looks as follows [6]:

$$
\left[\hat{\mathbf{R}}_{i j}\right]-\frac{R}{2}\left[\hat{\mathbf{g}}_{i j}\right]+\Lambda\left[\hat{\mathbf{g}}_{i j}\right]=\frac{8 \pi G}{c^{4}}\left[\hat{\mathbf{T}}_{i j}\right]
$$

where $\left[\hat{\mathbf{R}}_{i j}\right]$ is the Ricci curvature tensor obtained from the Riemannian curvature tensor $\left[\hat{\mathbf{R}}_{i j k m}\right]$ by means of convolving with its pair of indices; $R$ is the scalar curvature, i.e. the convolved Ricci curvature tensor; $\left[\hat{\mathbf{g}}_{i j}\right]$ is the metric tensor; $\Lambda$ is cosmological constant; $\left[\hat{\mathbf{T}}_{i j}\right]$ is the stress-energy-momentum tensor; $c$ is the speed of light in vacuum; $G$ is the gravitational constant.

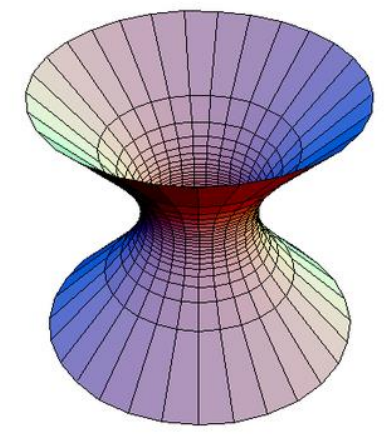

Fig. 3. Graphical interpretation of the Schwarzschild solution. 
Einstein's equations do not impose any constraints on use to describe the «space-time» coordinates, i.e. have the property of general covariance and limit the choice of only 6 of the 10 independent components of a symmetric metric tensor. Therefore, their decision is ambiguous without introducing some constraints on the metric components, called the coordinate conditions [7]. Solving Einstein's equation (1) in conjunction with properly chosen coordinate conditions, you can find all the 10 independent components of the symmetric metric tensor.

The metric tensor makes it possible to determine the square of the interval in curved space that defines the distance in a metric space:

$$
\delta S^{2}=\left[\hat{\mathbf{g}}_{i j}\right](x) \delta x^{a} \delta x^{b} .
$$

Consider separately the components of the equation (1). This equation assumes four-dimensional space-time, so it is considered its components in four-dimensional space. According to [8], in the so-called Schwarzschild coordinates $t, r, \theta, \varphi$, the last 3 of which are similar to spherical, the metric tensor is of the form:

$$
\left[\hat{\mathbf{g}}_{i j}\right]=\left[\begin{array}{cccc}
1-\frac{r_{s}}{r} & 0 & 0 & 0 \\
0 & -\left(1-\frac{r_{s}}{r}\right)^{-1} & 0 & 0 \\
0 & 0 & -r^{2} & 0 \\
0 & 0 & 0 & -r^{2} \sin ^{2} \theta
\end{array}\right],
$$

Where $r_{s}$ is the Schwarzschild radius equal to the gravitational radius.

Expression (2) in this metric is written as follows:

$$
\delta S^{2}=\left(1-r_{s} r^{-1}\right) c^{2} \delta t^{2}-\frac{\delta r^{2}}{1-r_{s} r^{-1}}-r^{2}\left(\sin ^{2} \theta \delta \varphi^{2}+\delta \theta^{2}\right)
$$

In the fig. 3 there is a graphical interpretation of the space by Schwarzschild. On the basis of similarity in fig. 2 and fig. 3 , it can be concluded on the applicability of the foregoing mathematical apparatus for the construction of strictly equal-contrast color space.

The next element of the equation (1) is the stress-energy tensor, which in our case will be replaced in the future on the color energy tensor.

The curvature of color discrimination space. Now consider the concept of the curvature of color discrimination space, which can be described by the Ricci tensor. A scalar value can be built from it by the following formula:

$$
R=\sum_{i=1}^{2} \sum_{j=1}^{2}\left[\hat{\mathbf{R}}_{i j}\right]\left[\hat{\mathbf{g}}_{i j}\right]
$$

The transition from the components of the Ricci tensor to the scalar curvature $R$ is, at first sight, loss of information, i.e. nine variables are replaced by one. However, in the two-dimensional case, no data loss occurs. Indeed, the components of the curvature tensor are skew-symmetric both in the upper pair of indices and in the lower. In the paper there is proved that in the case of the spherical surface with radius $r_{0}$ the scalar curvature is calculated as $R=2 / r_{0}$.

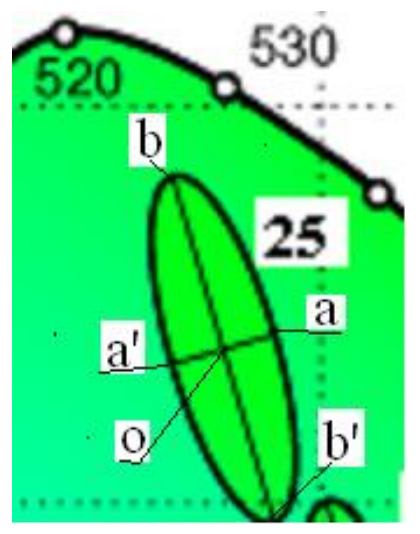

Fig. 4. Separate MacAdam ellipse. 


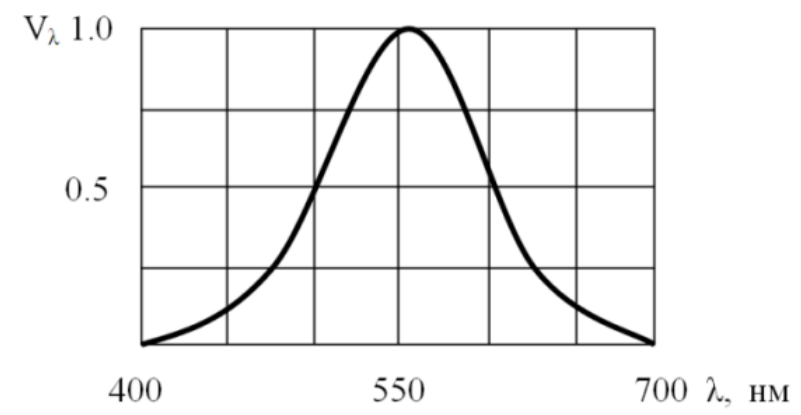

Fig. 5. Visibility curve.

Color energy tensor. Introduce the concept of color energy tensor $\left[\hat{\mathbf{C}}_{i j}\right]$. For new components of this tensor, turn to fig. 4. Evidently, the energy density in the stress-energy tensor in general relitivity will correspond to the value of the MacAdam ellipse brightness density. According to MacAdam, the ellipse and in view of brightness - an ellipsoid is a threshold of color discrimination and brightness. Thus, from the viewpoint of the eye, this ellipsoid will be perceived as a geometrical point, there will be no color and brightness differences in the field (and also inside it). Therefore, the light energy density is equal to the brightness of the point, for example, in the center of the ellipse (fig. 4). Since these ellipses MacAdam received in his experiments (measuring color coordinates) in the first half of the last century, and in fact nowadays it is rather difficult to repeat these experiments, so the application has been developed, that allows determining the coordinates of any point and its brightness by the image of the ellipses on the color locus. To determine the brightness of these points we proceed as follows. Assume that the color of every point of the ellipse is created using monochrome emitters (two spectral colors).In calculating the brightness of the spectral colors a visibility curve can be taken (fig. 5) and in accordance with the spectral color wavelength of the color locus it can be determined the relative brightness sensation value on this curve.

Passing the math, due to their bulkiness, give the final expression for the color energy tensor:

$$
\left[\hat{\mathbf{C}}_{i j}\right]=\left[\begin{array}{cccc}
L_{0} & L_{d} & L_{a} & L_{b} \\
L_{d} & \left(L_{d}-L_{0}\right) S^{-1} & \left(L_{a}-L_{0}\right) S^{-1} & \left(L_{b}-L_{0}\right) S^{-1} \\
L_{a} & \left(L_{d}-L_{0}\right) S^{-1} & \left(L_{a}-L_{0}\right) S^{-1} & \left(L_{b}-L_{0}\right) S^{-1} \\
L_{b} & \left(L_{d}-L_{0}\right) S^{-1} & \left(L_{a}-L_{0}\right) S^{-1} & \left(L_{b}-L_{0}\right) S^{-1}
\end{array}\right],
$$

where $S$ is the ellipse area; $L_{0}, L_{a}, L_{b}, L_{d}$ are the radiance of the ellipsoid center and the points on the ellipsoid surface corresponding to the main axes.

Also, introducing the amount of color discrimination threshold $r_{0}$ to the elements of the metric tensor (3), get:

$$
\left[\hat{\mathbf{g}}_{i j}\right]=\left[\begin{array}{cccc}
1-\frac{r_{0}}{r} & 0 & 0 & 0 \\
0 & -\left(1-\frac{r_{0}}{r}\right)^{-1} & 0 & 0 \\
0 & 0 & -r^{2} & 0 \\
0 & 0 & 0 & -r^{2} \sin ^{2} \theta
\end{array}\right] .
$$

Thus, the transition to strictly equal-contrast color system reduces to solving equations of the form similar to equation (1):

$$
\frac{2}{r_{0}}[\hat{\mathbf{I}}]-\frac{1}{r_{0}}\left[\hat{\mathbf{g}}_{i j}\right]=\left[\hat{\mathbf{k}}_{i j}\right]\left[\hat{\mathbf{C}}_{i j}\right],
$$

where $\left[\hat{\mathbf{k}}_{i j}\right]$ is the diagonal matrix of constants that are proportional to coefficients of frame «mobility» in the space of Riemann geometry; $\hat{\mathbf{I}}$ is the unit diagonal matrix, $4 \times 4$ dimension; color energy tensor and the metric tensor are defined by equations (6) and (7).

Thus, the transition to a strictly equal-contrast color space system is reduced to the determination of the tensor elements $\left[\hat{\mathbf{k}}_{i j}\right]$. Of the article scope limitation there is omitted the math and written the expressions for the coefficients:

$$
\begin{aligned}
& k_{11}=\frac{\left(2-r_{0} b^{2} d^{2}\right) \zeta_{\mathrm{T}}}{r_{0}} ; \\
& k_{22}=\frac{\left(2-r_{0}^{3} a^{2} d^{2}\right) \zeta_{\mathrm{H}}}{r_{0}} ; \\
& k_{33}=\frac{\left[2-r_{0}^{3} a^{2} b^{2}\left(1-d^{2}\right)\right] \sin ^{2} \theta L_{S}}{r_{0}},
\end{aligned}
$$


Image Processing, Geoinformation Technology and Information Security / L.D. Lozhkin, O.V. Osipov

where $a, b, d$ are the ellipsoid semi-axes; $\zeta_{\mathrm{H}}$ is the color saturation; $\zeta_{\mathrm{T}}$ is the color hue; $L_{S}$ is the brightness on the ellipsoid surface.

Thus, the relations (9), as measured the color saturation, the hue and the linear ellipsoid dimensions, allow the transition to a strictly equal-contrast color space.
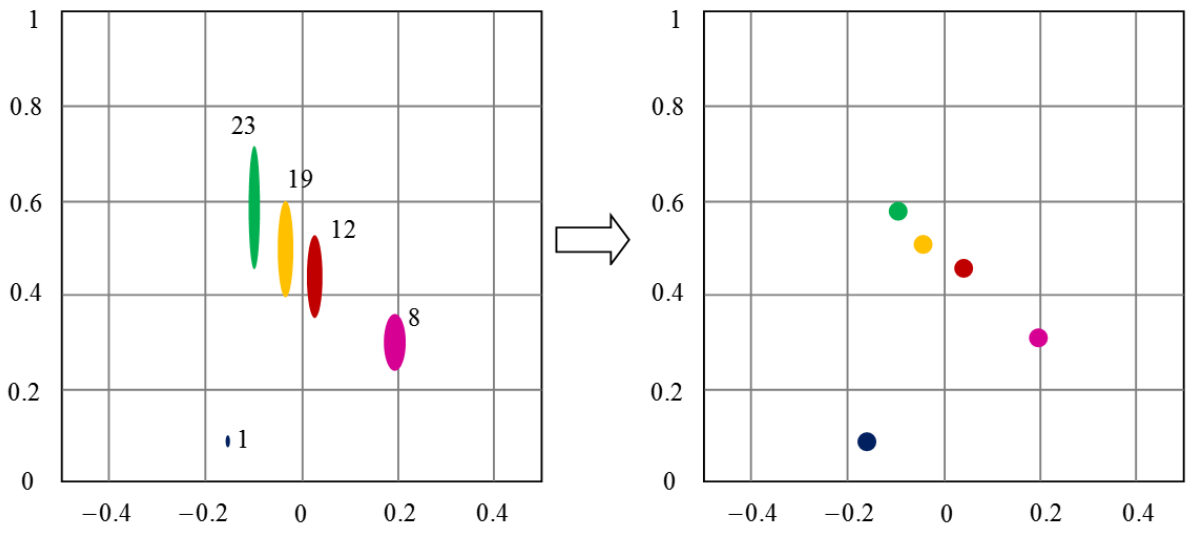

Fig. 6. Cross-section of ellipsoids and their transformation into equal size balls. The size of the ellipsoids and spheres is increased 10 times.

\section{Results and Discussion}

In fig. 6 and fig. 7 there are presented results of the transformation using the relations (9), taking into account the above mathematics. This transformation was carried out for the CIE 1931 colorimetric system $\left(x^{\prime}, y^{\prime}\right)$.

Obviously, using the above method it may be converted the color space of the Riemann space. For example, if it is depicted a sphere with radius equal to the maximum brightness (white color) for the CIE 1931 system $\left(x^{\prime}, y^{\prime}\right)$, and the color locus is drawn on the sphere surface, keeping the transition from the metric flat space to the Riemann space, using the basis of the moving frame, you can get a curved color space, which can be depicted in the same basis of the moving frame of color discrimination space (MacAdam balls).
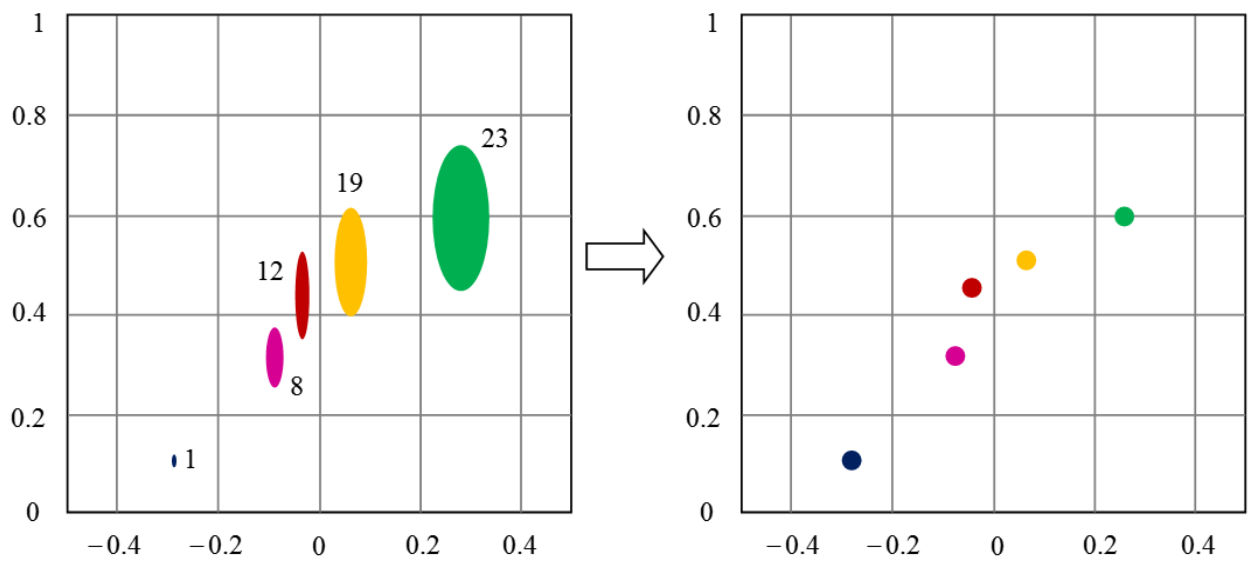

Fig. 7. Cross-section of ellipsoids and their transformation into equal size balls. The size of the ellipsoids and spheres is increased 10 times.

With this mathematical approach the input data can be presented in any of the existing CIE colorimetric systems such as CIE $1960(u, v)$ and (or) in CIE 1976 (Lab).

\section{Conclusion}

In summary, there are formulated the main conclusions of the work.

1. It has been found the similarity between space-time state and the color space.

2. On the basis of this similarity for developing the strictly equal-contrast color space it were used the Einstein's field equations.

3. The solution of these equations was carried out in four-dimensional space, which used three-dimensional metric space ( $u, v, w)$ in the CIE 1960 system and the fourth dimension was brightness, thereby creating the strictly equal-contrast fourdimensional color space. Similarly, the calculation for the three-dimensional color space was made.

4. It is shown that as the input color space can be used any of the known color spaces and it will be obtained the equal spheres of color discrimination thresholds at the output.

5. The resulting color body is a sphere which radius depends on the exact point representing the color. 
6. The color difference between two colors resulting in the strictly equal-contrast color system is determined by the length of the arc, linking two colors that lie on different surfaces of concentric spheres.

\section{References}

[1] Newhall SM. The ratio method in the review of the Munsell colors. Am. J. Psychol. 1939; 52: 394 p.

[2] Munsell A, Sloan T, Godlove I. Neutral value scales I. Munsell neutral value scale. Opt. Soc. Am. 1933; 23: 394 p.

[3] Jimenez JR, Hita E, Romero J, Jimenez L. Scalar curvature of space as a source of information of new uniformity aspects concerning to color representation systems. J. Optics 1993; 24(6): 243-249.

[4] Mac Adam DL. Visual sensitivities to color differences. Josa 1943; 33(18).

[5] Mac Adam DL. Color essays. Josa 1975; 65(5): 463-485.

[6] Pauli W. Theory of relativity. Ed. by Ginzburg VL and Frolova VP. M.: Nauka, 1991; 328. (in Russian)

[7] Fock VA. Theory of space, time and gravity. M.: GITTL, 1955; 504 p. (in Russian)

[8] Schwarzschild K. About the gravitational field of a mass point in the Einstein's theory. In: Albert Einstein and the gravitational theory. M.: Mir, 1979; 199207. (in Russian) 